\title{
Experimental evidence of non-classical brain functions
}

\author{
Christian Matthias Kerskens ${ }^{{ }^{*}}$ and David López Pérez ${ }^{1,2}$ \\ ${ }^{1 *}$ Trinity College Institute of Neuroscience, Trinity College \\ Dublin, Lloyd Institute, Dublin, 2, Republic of Ireland. \\ ${ }^{2}$ Institute of Psychology, Polish Academy of Sciences, Jaracza 1, \\ Warsaw, 00-3787, Poland.
}

*Corresponding author(s). E-mail(s): christian.kerskens@tcd.ie; Contributing authors: david.lopez@psych.uw.edu.pl;

\begin{abstract}
Exploring unknown quantum systems is an experimental challenge. Recent proposals exploring quantum gravity have suggested circumventing this problem by considering the unknown system as a mediator between two known systems. If such a mediation can locally generate entanglement in the known systems, then the mediator must be nonclassical.

The same approach may be applicable to other systems, in particular the brain, where speculations about quantum operations in consciousness and cognition have a long history. Translated to the brain, the mediator is then an unknown brain function. For the quantum systems, we could use proton spins of bulk water, which most likely interfere with the any brain function. Entanglement in these spins can be witnessed with multiple quantum coherence (MQC). We based our witness protocol on zero quantum coherence (ZQC) whereby potential signals from local properties were minimised.

For short repetitive periods, we found ZQC signals in large parts of the brain, whereby the temporal appearance resembled heartbeatevoked potentials (HEPs). Similar to HEPs, we also found that the ZQC signal depended on conscious awareness. Consciousnessrelated signals have, to our knowledge, not yet been reported in NMR. Remarkably, we could exclude local properties as contrast mechanism because (a) the ZQC signals had no correlates known in conventional MRI, and (b) the ZQC signals only appeared if
\end{abstract}


the local properties of the magnetisation, which are complementary to non-local properties, were reduced. Our findings suggest that we may have witnessed entanglement mediated by consciousnessrelated brain functions. Those brain functions must then operate non-classically, which would mean that consciousness is non-classical.

Keywords: quantum brain, MRI, entanglement witness, heartbeat evoked, zero quantum coherence

\section{Introduction}

The idea that the mind may be connected with quantum mechanics has a long history [1]. Despite heated discussions and speculations [2-7], experimental evidence for the quantumness of the brain was only found over a decade ago. Surprisingly, non-physical experiments discovered those breakthroughs. Instead, the analysis of psychological observations have shown that the brain may use non-classical operations $[8,9]$.

This new promising field of quantum psychology bases the analysis entirely on quantum mechanics, which contrasts with computational Neuroscience. Computational Neuroscience is founded on the physiology of single brain cells and networks, which operate in time and space domains far beyond Planck's constant. As such, experimental results in the field which have been collected over many years are all classical. Consequently, computational neuroscience, which intends to simulate the outcome of brain computation, is entirely classical.

Contradictorily, the quantum supremacy principle [10] says, that classical computation, be it by a computer or a brain, would be unable to simulate quantum mechanics in any realistic timeframes. For computational neuroscience, that means that it can't simulate the outcome of (quantum) psychology experiments nor explain how the brain computes; it fails this essential test.

To overcome this paradox, we hypothesise the existence of an unknown biological quantum (field) system, which may extend far beyond Planck's constant allowing quantum computation in single cells and even cell networks. Such a quantum system could then explain the classicality of experimental results in physiology ${ }^{1}$ as well as the quantumness of psychology.

To test our hypothesis, we implemented an approach which is based on recent proposals in quantum gravity $[12,13]$, where it was suggested circumventing the lack of a theory by using the graviton as a mediator between two quantum systems. It was shown then that if a mediator can create entanglement between the two quantum systems, then the mediator itself must be non-classical. We applied this nice idea here.

Translated to the brain, the mediator is then an unknown brain function. For the quantum systems, we used the proton spins of bulk water in the brain,

\footnotetext{
${ }^{1}$ The lack of an adequate theory may hinder the development of appropriate instrumentations to measure quantum effects which then is the reason the view on quantum biology in the brain is obstructed or classical[11].
} 
which most likely interfere with the any brain function. If the proton spins entangled during brain activity, then we would know that a non-classical brain function must exist.

Therefore, we only have to witness that brain functions can produce entanglement in the auxiliary systems of the proton spins.

We used an MRI sequence that could contain zero quantum coherences (ZQC) as entanglement witness for the proton spins. It has previously been shown that an increase in the signal amplitude in any multiple quantum coherence (MQC) is a witness [14].

Unfortunately, a MQC signal increase in biological systems is inconclusive because the signal of MQC may also vary due to rotational symmetry breaking or susceptibility changes [15-18]. To avoid misinterpretation, we designed the sequence in a way, that any possible signal variation based on those mechanisms were at noise level, and therefore not detectable.

At first sight, the use of a noisy sequence seems contra-productive in the attempt to find improbable occurrences. However, macroscopic quantum complementarity relations between local and non-local properties suggest that local properties have to be suppressed to allow the detection of non-localities $[19,20]$, which in MRI is mostly avoided as it would result in low signal in every MRI technique.

The aim of our study was to find evidence of non-classicality. As pointers for brain computation, we focussed on electrophysiological brain waves which are presented in the conscious aware brain at rest.

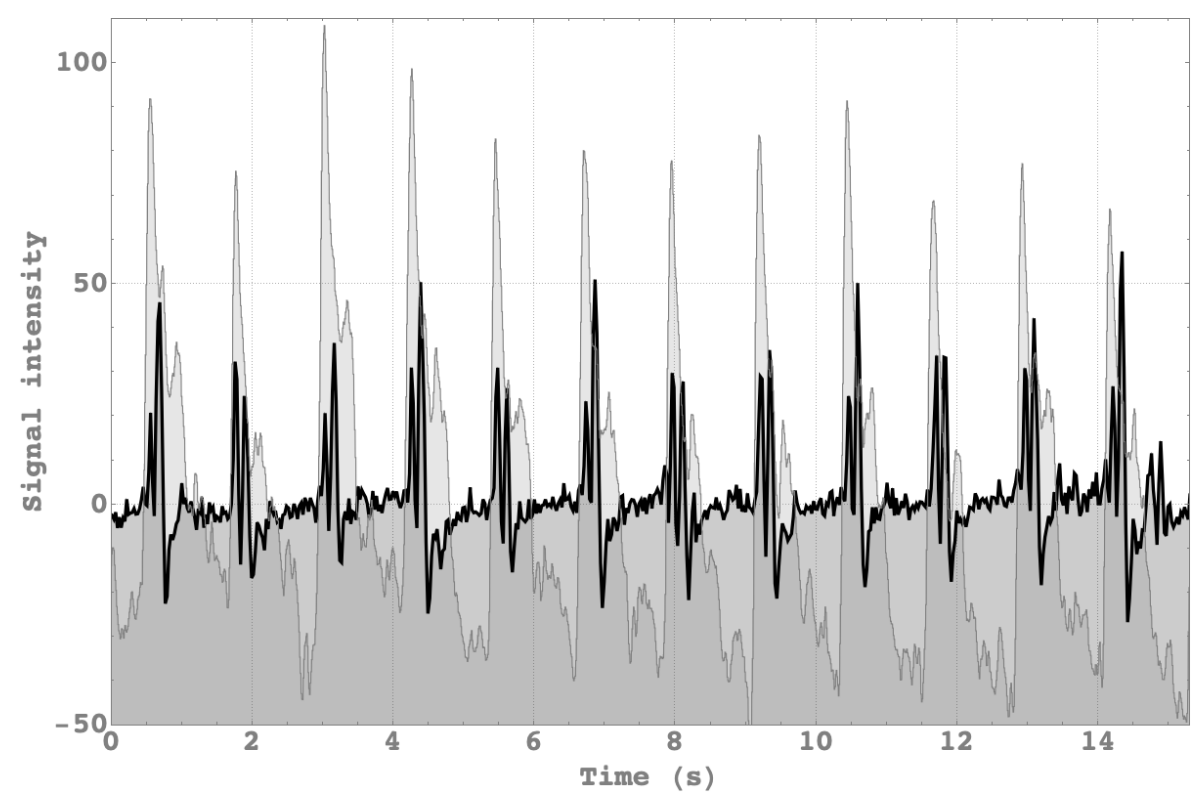

Fig. 1 MRI signal time course (Black) during 12 heart cycles compared with simultaneous oximeter reading of a finger (Grey). 


\section{Results}

We used the EPI time series 3 in human volunteers at rest. The beginning of the sequential RF-pulses train of the EPI time series were used to saturate the magnetisation of the imaging slice. The desired reductions of the local NMR component were normally reached shortly before the equilibrium magnetisation.

Then, we found regular, repeating signal bursts of predominant signal alternations in single volumes of the brain slices as shown in Fig. 1, where the signal peaks of the bursts increased by up to $15 \%$. In most cases, the alteration was sequential from one image acquisition to the next.

In the following, we will focus on the NMR contrast mechanism of the signal first, and then how it related to physiology and mind.

\subsection{NMR contrast}

The burst signal had the following properties;

The burst signal alternated during burst which confirmed that at least two RF pulses were necessary to generate the signal. The two RF-pulses always enwrap an asymmetrical gradient interval $G_{a} T_{a}$ (Fig. 5), which is the basic pulse design to measure ZQC. The consequential long-range ZQC contrast was verified further by altering sequence parameters.

For rotating the asymmetric gradients $G_{a}$, we found the characteristic angulation dependency of the dipole-dipole interaction as shown is (Fig. 2A). The plot represents the fitted function $\left|\left(3 \cdot \cos ^{2}[\varphi]-1\right)\right|$ (adjusted $\mathrm{R}^{2}$ test of goodnessof-fit resulted in $\mathrm{R}^{2}=0.9958$ ) where $\varphi$ takes the additional gradients in read and phase direction into account. At the magic angle, the burst signals disappeared.

For the flip angle variation, we found the predicted signal course for the ZQC flip angle dependency [21] which was fitted to the data $\left(\mathrm{R}^{2}=0.9964\right)$. Predicted maximum at $45^{\circ}$ could be confirmed (Fig 2B). In contrast, the Ernst-angle [22] which is a good indication for the optimum angle for SQC is around $13^{\circ}$ (for $\mathrm{T}_{1}=1.5 \mathrm{~s}$ ).

For the alteration of the off-resonance frequency, we found a typical magnetisation transfer contrast (MTC) change for the baseline which depended on the off-resonance frequency (Fig. 2C). In contrast, the signal intensity showed the remarkable immunity to MTC as expected for ZQC [23] with no significant changes in the same frequency range (Fig. 2D).

The effects of the competing effects, the build up of the ZQC on the one hand and de-phasing over time on the other hand, were studied varying the TR. We found that from $38 \mathrm{~ms}$ onwards the signals showed no growth of ZQC. The free induction dominated.

Finally, we varied the slice thickness to study Time-of-flight effects. We found no significant influence on the relative signal. 
(A)

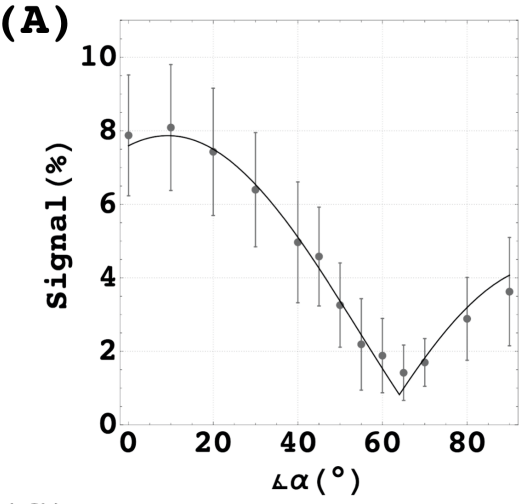

(C)

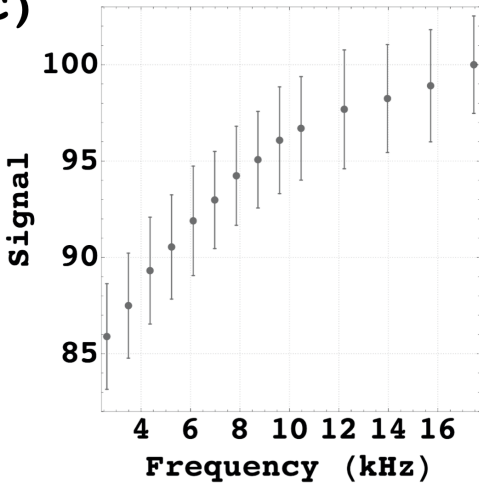

(E)

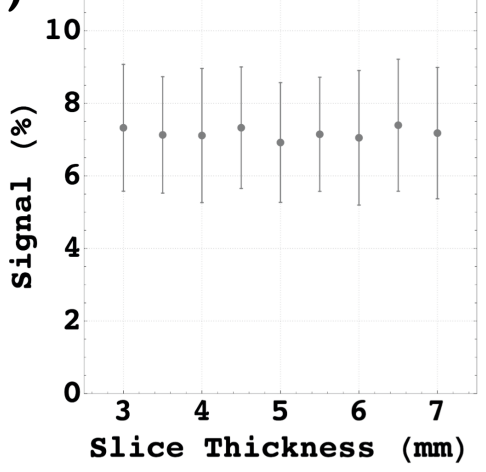

(B)

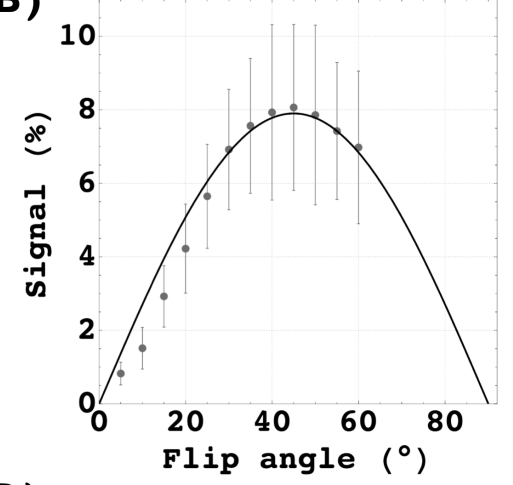

(D)

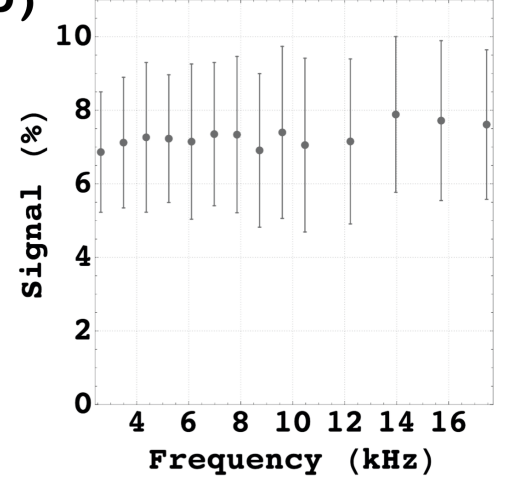

(F)

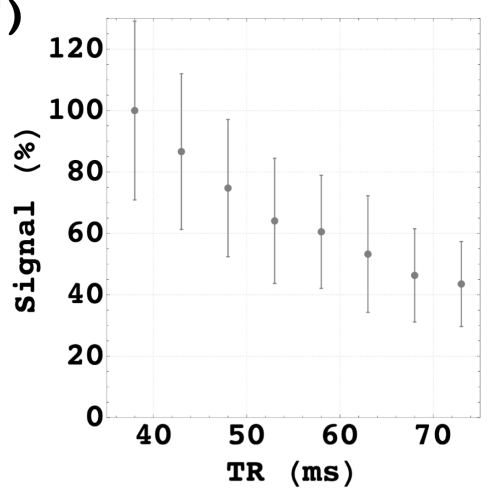

Fig. 2 Variation of sequence parameters. Data shows signal averaged over 5 subjects. Error bars represent the standard deviation from the mean. (A) Signal intensity plotted against the slice gradient angulation $\alpha$ in respect to the magnetic main field. (B) Signal plotted against flip angle variation. ZQC prediction plotted in Black. (C) Signal intensity plotted against the frequency offset of the saturation slices of the BS and (D) averaged signal of the AMP. (E) Relative signal change plotted against slice thickness. (F) Signal plotted against repetition time. 

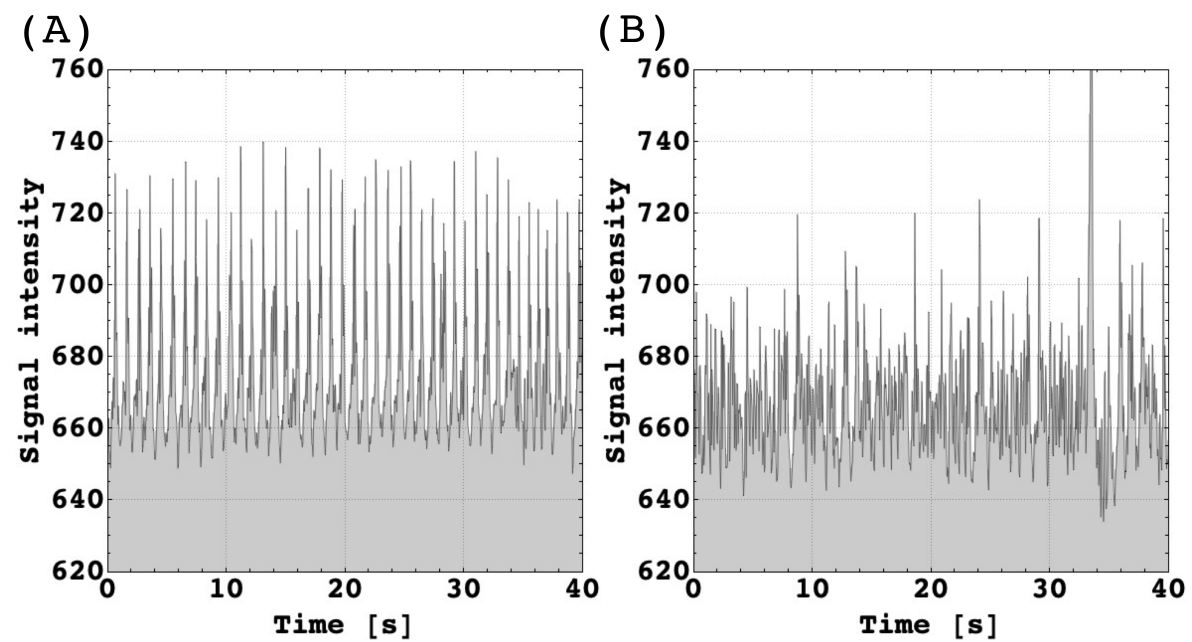

Fig. 3 Pattern observed in participant who had reported falling asleep. (A) Wake period. (B) Asleep, ZQC burst signals declined coincident with an increase of the S/N level. At $34 \mathrm{~s}$, the peak resulted from short head movement.

\subsection{Physiology and Mind}

The periods of signal bursts repeated with the same rate as the heart-beat. We used three temporal reference systems; (a) a finger pulse oximetry, (b) an electrocardiogram (ECG), and (c) the time-of-flight signal of a voxel placed in the superior sagittal sinus. The signal bursts appeared with the pulse from the finger pulse oximetry (Fig. 1). In relation to the ECG, we found using the Cross-Recurrence Quantification Analysis that the maximum burst signal was delayed by $0.3 \mathrm{~s}$ on average. With the start of the venous outflow, the bursts always ended as shown in Fig. 4 and Fig. A3.

Regarding the duration of the bursts under normal conditions, we mostly observed two sequential peaks which equaled 4 TRs adding up to a time period of $180 \mathrm{~ms}$. We also saw longer periods building up to 10 TRs (see Fig. 4B) extending the period to $450 \mathrm{~ms}$.

We located the bursts in brain tissue of all slices except around the periventricular area (probably due to movement induced by ventricular pulsation in those regions [24]) as illustrated in Fig. A2.

The global aspect conformed with another interesting feature; the signal could be restored while being averaged over the entire tissue component of the imaging slice (Fig. 1 and Fig. 4B, single voxel time course are shown in Fig. A1).

We also found that the signal did not respond to the $\mathrm{CO}_{2}$ challenge (Fig. 4B) in contrast to the SQC signal from the voxel including the superior sagittal sinus (Fig. 4A) which indicated the blood flow response.

During our studies, we also realised that the signal depended on awareness and awakening. In seven participants from whom two had reported to have fallen 
asleep, we found that the signal pattern declined as shown in Fig. 3. For the final data acquisition, all participants had been asked to stay awake during the imaging protocol. At this point, we no longer detected a sleep pattern.

In a case study, we observed the pattern change over a period of 20 minutes which showed a gradual transition from awake to asleep as shown in the appendix at Fig. A4.

We used Recurrence Quantification Analysis and Multifractal Detrended Fluctuation Analysis to illustrate the difference between wakefulness and the slow decline during the falling asleep period. The analysis shows that periodicity, large and small fluctuations, repeating patterns and their predictability, and the stability of the system were changing over the observation period (Fig. A5).

\section{Methods}

We studied 40 subjects (between 18 and 46 years old) using a 3 Tesla wholebody MRI scanner (Philips, The Netherlands) which was operated with a 32-channel array receiver coil.

Imaging protocols were approved by Trinity College Dublin School of Medicine Research Ethics Committee.

All participants of final data acquisition were asked to stay awake and stay still during the imaging protocol, or to report any failure to do so.

Fast gradient-echo EPI (GE-EPI) time series were carried out which had been optimised over a wide range of participants. The finalised parameters were as follows: $\mathrm{FA}=45^{\circ}, \mathrm{TR}=45 \mathrm{~ms}, \mathrm{TE}=5$, voxel size $=3.5 \times 3.5 \times 3.5 \mathrm{~mm}$, matrix size $=64 \times 64$, SENSE factor $=3$, bandwidth readout direction $=2148$ $\mathrm{Hz}$, saturation pulse thickness $/$ distance $=5 / 20 \mathrm{~mm}$.

Two saturation pulses placed parallel to the imaging slice (Fig. 5) were added which allowed us to vary long-range correlation of the ZSE and MTC. Saturation gradients had a time integral (length $\mathrm{x}$ strength) of $\mathrm{GT}_{s}=5.1 \mathrm{~ms} \times 6.25$ $\mathrm{mT} / \mathrm{m}$, the crusher gradients in read and slice direction of $\mathrm{GT}_{c}=1.3 \mathrm{~ms} \mathrm{x}$ $25 \mathrm{mT} / \mathrm{m}$, the slice rephase gradient of $\mathrm{GT}_{r}=0.65 \mathrm{~ms} \times 25 \mathrm{mT} / \mathrm{m}$, and the slice termination gradient of $\mathrm{GT}_{t}=0.65 \mathrm{~ms} \times 15 \mathrm{mT} / \mathrm{m}$. Gradients timing and arrangements are shown in Fig. 5. Gradients relevant for ZSE are shown in the asymmetry field and are marked with indices t, c, r, and s for identification. We rotated the asymmetric gradients in respect to the magnet field starting from coronal $0^{\circ}$ to axial $90^{\circ}$ in twelve steps; slice angulation $\alpha$ related to the angulation from the spin-spin interaction as

$\varphi=\alpha-\tan ^{-1}\left(\left[\mathrm{GT}_{c}-\mathrm{GT}_{r}\right] /\left[2 \cdot \mathrm{GT}_{s}+\mathrm{GT}_{c}+\mathrm{GT}_{t}\right]\right)=\alpha-9.6^{\circ}$. Further, we varied the correlation distance via altering the amplitude and the duration of the saturation gradients.

We also altered the following sequence parameters in pseudo-randomised orders:

(a) variation of the flip angle from $5^{\circ}$ to $60^{\circ}$ in steps of $5^{\circ}\left(60^{\circ}\right.$ was the power limit by the specific absorption rate (SAR)).

(b) the off-resonance frequency was varied as $[2.62,3.49,4.36,5.23,6.11,6.98$, 
7.84, 8.73, 9.60, 10.47, 12.22, 13.96, 15.71, 17.45] kHz.

(c) slice thickness from $3 \mathrm{~mm}$ to $7 \mathrm{~mm}$ in steps of $0.5 \mathrm{~mm}$.

(d) repetition time (TR) varied from $38 \mathrm{~ms}$ to $73 \mathrm{~ms}$ in steps of $5 \mathrm{~ms}$.

Further, we explored the signal distribution over the entire brain. 9 slices (in 5 volunteers) were acquired at different positions, with slices from bottom to the top covering all anatomical regions.

In a breath-holding challenge, four participants were asked to stop breathing for $20 \mathrm{~s}$ without taking a deep breath. Body movements were reduced through multiple cushions immobilizing the head.

For the time reference analysis, we used Cross-Recurrence Quantification Analysis [25] to calculate the delay between the R-wave in electrocardiogram (ECG) and the MRI signal. For the calculation, we used the CRP Toolbox [26, 27] for Matlab [28].

For the NMR contrast analysis, we used the averaged maximum peak of the burst and the signals between bursts as baselines. Calculations were performed using the routine by Gomes et al. [29] which was implemented in Matlab [28]. Preprocessing included the following; Rescaling, which was applied to all data sets before any analysis using the MR vendor's instructions. Visual inspection of average time series in search for irregularities which were manually removed from the analysis leaving the rest of the time series unaltered. Manual segmentation was used to create a mask to remove cerebral spinal fluid (CSF) contributions. The first 100 of 1000 scans were removed to avoid signal saturation effects. The manual segmentation of the masks was eroded to avoid partial volume effects at the edges.

For the analysis of sleeping pattern, we used a Recurrence Quantification Analysis and a Multifractal Detrended Fluctuation Analysis (for detailed description see Lopez-Perez et al. [30]).

All data graphics were created with Mathematica [31]. Data and source code for analysis are available at www.github.com/Mirandeitor/Entanglementwitnessed-in-the-human-brain.

\section{Discussion}

Experimentally, we aimed to prove that brain functions can create entanglement in auxiliary quantum system.

Thereby, we employed a hybrid MRI sequence which could contain SQC and ZQC, simultaneously. We found signal burst with every heartbeat where we were able to show in section 2.1 that the underlying contrast mechanism was ZQC. Therefore, it is possible that we witnessed quantum entanglement.

However, the increase of $\mathrm{ZQC}$ alone is not a sufficient condition for witnessing entanglement in biology because ZQC is altered by many other physiological changes, too.

Ultimately, we have to exclude that the signal bursts were caused by a "classical" zero quantum coherence. That's why the following discussion will focus on the incompatibility of classical mechanisms. 
First, we would like to remark that ZQC can be altered by local fieldinhomogeneities and anisotropies [15-18]. Those changes in ZQC have complementary contrasts in SQC, namely $\mathrm{T}^{*}$ relaxation and diffusion, which both alter during the heart cycle.

However, $\mathrm{T}^{*}$ changes have shown a different temporal (shifted by more than half of the cycle time in respect to the ZQC signal) and spatial response (higher signal at blood vessel) [32]. The tissue response at around $2 \%$ is much lower than during functional activation. In contrast, functional activations showed no significant changes in the ZQC burst signal and only minimal signal increases at the baseline [33]. Therefore, we can conclude that local field-inhomogeneity changes are ineligible as a signal source.

Besides local field-inhomogeneity, ZQC depends on order [34] and rotational symmetries $[15,16]$ which can be probed with diffusion MRI. The order may correlate with the apparent diffusion coefficient (ADC), while the fractional anisotropy (FA) indicates the rotational symmetry breaking. In praxis, MQC signals are higher at decreased ADC and increased FA.

Nakamura et al. [35] have shown that the temporal changes of the ADC are in phase with the intracranial volume change while FA is shifted by $180^{\circ}$. Our ZQC signals coincided with the transition phase from the highest to the lowest ADC (and vice versa for the FA). From those results, we can conclude that the theoretical optimum (ADC minimal, FA maximal) for a classical ZQC is outside the time window of the ZQC bursts.

As we have mentioned before, our sequence design had a poor S/N. Therefore, it wasn't surprising that the sequence couldn't pick up changes for which, theoretically, ZQC should be sensitive for, like diffusion and field-inhomogeneities changes. For "classical fluids", however, the signal-to-noise ratio $\mathrm{S} / \mathrm{N}$ in MQC is much lower than in SQC. For example, the S/N of ZSE compared to the conventional MRI signal (SQC) only reaches up to 0.05 at 4 Tesla, experimentally [36, 37]. Our sequence was suboptimal because we replaced a $90^{\circ}$ by a $45^{\circ}$ RF-pulse (reduction by factor 2), used a 3 Tesla field, and the evolution time was shorter. Therefore, we could conclude that in combination with the EPI readout ZQC signals were negligible.

In this estimation, we considered the maximal signal amplitude, not fluctuations of it. Translated to a conventional ZQC, the burst would be serval magnitudes higher than the averaged ZQC signal, which has never been reported.

Although, we found that the bursts have no correlates in SQC imaging, the correlation with the cardiac cycle suggested a relation with flow and motion effects which we further investigated.

We varied slice thickness and TR as possible sequence parameters, which are sensitive to time-of-flight effects. For the slice thickness, the relative signal did not vary significantly (Fig. 2E), for the repetition time, we found the free induction decay dominating the decline (Fig. 2F). Furthermore, when we varied the blood flow with the help of a $\mathrm{CO}_{2}$-challenge (Fig. 4), we found no significant response of the burst signal amplitude. 
Further, the fact the signal bursts have no significant SQC component (Fig $2 \mathrm{~A}$ at the magic angle), we can in principle exclude all SQC contrast mechanism including changes in $\mathrm{T}_{1}$ and $\mathrm{T}_{2}$ relaxation, line narrowing, or magnetic field shifts.

Above, we have established that conventional MR sequences, be it SQC or MQC, won't detect the observed signal bursts. Our sequence shouldn't detect it either.

However, in our sequence, the number of spins aligned along the same direction was reduced which changed the ratio of the partial quantum information shared between local properties $P$ and non-local properties $Q$ of spins. One has $Q+P \leq 1$ with $P=1$ for aligned spins [19]. Only with $P \ll 1$, the mediator could create non-local properties $Q$.

In summary, we found ZQC signals only if local properties $P$ were reduced. At the same time, the ZQC signals have no correlate in conventional MRI, be it in SQC or conventional MQC, which underpins that the complementarity is a necessary condition. Consequently, the signals had non-local properties.

It is important to remember that our findings can't have any physiological or cognitive relevance. They can only exist if local properties $P$ are reduced.

Otherwise, the existence of a biological mediator which can create entanglement is rather unexpected. From a mediator with such phenomenal properties, we would expect physiological or cognitive relevance.

A closer look at the signal behaviour confirms this assumption. We found that the timing of the signal bursts coincided with an electrophysiological event, which is known as heartbeat evoked potentials (HEP). The bursts also showed similar sensitivity to conscious awareness as HEP signals in the same time window [38].

In another study [30], López Pérez et al. have shown that the complexity of burst signals also correlate with psychological test results in short-term memory.

Lastly, we would like to discuss why the signals only appeared during this very specific time window. How would this fit into a quantum brain theory?

We would like to propose a possible explanation as follows; If the brain were quantum, then it may work at different scales from cell organelles, cells to cell networks. Such a scaling may explain why, for example, the memory system operates at different time scales from short to long term [39]. At some point, communication between these systems must be ensured. Let us now assume that the brain is genuinely quantum. If the brain was genuinely quantum at least for short periods, then quantum processes could communicate over all scales. Then (or maybe only then), it could also entangle auxiliary systems provided by an MRI sequence, which play otherwise no role in the physiological processing.

Although, we have now pushed the envelope even further, being genuinely quantum at some stage may be important to realise a quantum brain. Interestingly, genuinely quantum system are non-markovian and so far, the most promising models to create macroscopic quantum systems [11]. 
The genuinely quantum system during burst would explain why we saw no effects related to blood flow during the $\mathrm{Co}_{2}$ challenge because the system would be genuinely quantum independent of any blood flow changes, and therefore able to entangle our auxiliary system in any case (during consciousness).

Although, we couldn't find any blood flow effects, the relation with the heart beat suggest a hemodynamic origin of the phenomenon. Interestingly, the time window of the burst coincided with the period when the brain creates a reverse mechanism to transport tissue water back into the venous system. This reverse mechanism to maintain the balance between in- and outflow must then be non-markovian because it is a necessary condition for being genuinely quantum. The non-markovian mechanism, which may remember (or even know in advance) about the inflow, creates then an elusive blood wave traveling instantly from arterial to the venous blood system, despite the fact that those systems aren't connected and that the interconnecting volume between them, the tissue (through which the blood water can freely diffuse) and the capillaries, are by many magnitudes larger than the blood vessel volume.

The genuinely quantum hypothesis may explain why we were able to observe the mediation. More importantly, it may give an explanation why quantum biology beyond Planck's constant may only be observed if the system is undisturbed. In this case, that means alive and conscious. A situation which is difficult to achieve in biological studies which could be the reason nobody has observed similar results before.

\section{Conclusion}

The aim of this study was to show that the brain is non-classical. We assumed that unknown brain functions exist which can locally mediate the entanglement of auxiliary quantum systems. The experimental detection of such an entanglement created by the brain would then be sufficient to prove non-classicality. We found experimental evidence that such entanglement creation occurs as part of physiological and cognitive processes.

We argued that the ZQC signals are non-local because (a) ZQC can be an entanglement witness, (b) the signals have no SQC and $\mathrm{MQC}^{2}$ correlates,(c) they are only detectable if local properties are reduced, and (d) they resemble HEPs which are below verifiability in conventional MRI.

Our findings confirm that the mind operates non-classically, which seems like an obvious assumption considering what we know today about cognition and computing.

However, our findings are standing against the unproven statement that quantum entanglement or coherence can't survive in the hot and wet environment of the brain long and far enough.

Despite all controversy, we found an undiscovered NMR contrast, which can

\footnotetext{
${ }^{2}$ using the conventional MQC sequence design
} 
detect brain activity beyond conventional functional MRI. It may have interesting applications in psychology and medicine beyond the fundamental question we tried to answer here.

\section{Declarations}

- Funding

This research project was funded by Science Foundation Ireland from 201115 (SFI-11/RFP.1/NES/3051) and supported by Trinity College Institute of Neuroscience.

- Authors' contributions

Christian Matthias Kerskens: conceptualisation, methodology (physics), writing (original draft), supervision, funding acquisition.

David López Pérez: methodology (analysis), software, acquisition of data, data curation.

\section{Appendix A Extended Data}

\section{References}

[1] Atmanspacher, H.: Quantum Approaches to Consciousness. In: Zalta, E.N. (ed.) The Stanford Encyclopedia of Philosophy, Summer 2020 edn. Metaphysics Research Lab, Stanford University, ??? (2020)

[2] Penrose, R.: On the gravitization of quantum mechanics 1: Quantum state reduction. Foundations of Physics 44(5), 557-575 (2014). https://doi.org/ 10.1007/s10701-013-9770-0

[3] Hameroff, S., Penrose, R.: Orchestrated reduction of quantum coherence in brain microtubules: A model for consciousness. Mathematics and Computers in Simulation 40(3-4), 453-480 (1996). https://doi.org/10.1016/ 0378-4754(96)80476-9

[4] Koch, C., Hepp, K.: Quantum mechanics in the brain. Nature 440(7084), 611-611 (2006). https://doi.org/10.1038/440611a

[5] Dennett, D.C.: Consciousness explained. Philosophy and Phenomenological Research 53(4), 905-910 (1993). https://doi.org/10.2307/2108259

[6] Tegmark, M.: Importance of quantum decoherence in brain processes. Physical Review E 61(4), 4194-4206 (2000). https://doi.org/10.1103/ physreve.61.4194

[7] Kerskens, C.: From Cerebral Circulation to Quantum Consciousness. In: Quantum Biology, Interlaken (2019). The Science of Consciousness 
[8] Aerts, D., Aerts, S., Gabora, L.: Experimental Evidence for Quantum Structure in Cognition. In: Quantum Interaction, pp. 59-70. Springer, ??? (2009). https://doi.org/10.1007/978-3-642-00834-4_7. https://doi.org/10. 1007\%2F978-3-642-00834-4_7

[9] Bruza, P.D., Wang, Z., Busemeyer, J.R.: Quantum cognition: a new theoretical approach to psychology. Trends in Cognitive Sciences 19(7), 383-393 (2015). https://doi.org/10.1016/j.tics.2015.05.001

[10] Preskill, J.: Quantum computing and the entanglement frontier (2012)

[11] Milz, S., Egloff, D., Taranto, P., Theurer, T., Plenio, M.B., Smirne, A., Huelga, S.F.: When is a non-markovian quantum process classical? Phys. Rev. X 10, 041049 (2020). https://doi.org/10.1103/PhysRevX.10.041049

[12] Marletto, C., Vedral, V.: Gravitationally induced entanglement between two massive particles is sufficient evidence of quantum effects in gravity. Phys. Rev. Lett. 119, 240402 (2017). https://doi.org/10.1103/ PhysRevLett.119.240402

[13] Bose, S., Mazumdar, A., Morley, G.W., Ulbricht, H., Toro š, M., Paternostro, M., Geraci, A.A., Barker, P.F., Kim, M.S., Milburn, G.: Spin entanglement witness for quantum gravity. Phys. Rev. Lett. 119, 240401 (2017). https://doi.org/10.1103/PhysRevLett.119.240401

[14] Gärttner, M., Hauke, P., Rey, A.M.: Relating out-of-time-order correlations to entanglement via multiple-quantum coherences. Phys. Rev. Lett. 120, 040402 (2018). https://doi.org/10.1103/PhysRevLett.120.040402

[15] Bouchard, L.-S., Rizi, R., Warren, W.: Magnetization structure contrast based on intermolecular multiple-quantum coherences. Magn Reson Med 48(6), 973-979 (2002)

[16] Bowtell, R., Gutteridge, S., Ramanathan, C.: Imaging the Long-Range Dipolar Field in Structured Liquid State Samples. Journal of Magnetic Resonance 150(2), 147-155 (2001)

[17] Capuani, S., Alesiani, M., Branca, R.T., Maraviglia, B.: New openings for porous systems research from intermolecular double-quantum NMR. Solid State Nuclear Magnetic Resonance 25(1-3), 153-159 (2004)

[18] Bouchard, L.-S., Wehrli, F.W., Chin, C.-L., Warren, S.W.: Structural anisotropy and internal magnetic fields in trabecular bone: Coupling solution and solid dipolar interactions. Journal of Magnetic Resonance 176(1), 27-36 (2005) 
[19] Wieśniak, M., Vedral, V., Brukner, Č.: Magnetic susceptibility as a macroscopic entanglement witness. New Journal of Physics 7(1), 258 (2005). https://doi.org/10.1088/1367-2630/7/1/258

[20] Fan, X.-G., Sun, W.-Y., Ding, Z.-Y., Ming, F., Yang, H., Wang, D., Ye, L.: Universal complementarity between coherence and intrinsic concurrence for two-qubit states. New Journal of Physics 21(9), 093053 (2019). https: //doi.org/10.1088/1367-2630/ab41b1

[21] Zhong, C., Shaokuan, Z., Jianhui, Z.: Optimal RF flip angles for multiple spin-echoes and iMQCs of different orders with the CRAZED pulse sequence. Chemical Physics Letters 347(1), 143-148 (2001)

[22] Ernst, R.R., Anderson, W.A.: Application of Fourier Transform Spectroscopy to Magnetic Resonance. Review of Scientific Instruments 37(1), 93-102 (1966). https://doi.org/10.1063/1.1719961

[23] Uzi, E., Gil, N.: Enhancement of magnetization transfer effects by inter-molecular multiple quantum filtered NMR. Journal of Magnetic Resonance 190(1), 149-153 (2008)

[24] Nunes, R., Jezzard, P., Clare, S.: Investigations on the efficiency of cardiac-gated methods for the acquisition of diffusion-weighted images. J Magn Reson 177(1), 102-110 (2005)

[25] Marwan, N., Kurths, J.: Nonlinear analysis of bivariate data with cross recurrence plots. Physics Letters A 302(5), 299-307 (2002). https://doi. org/10.1016/S0375-9601(02)01170-2

[26] http://tocsy.pik-potsdam.de/CRPtoolbox/

[27] Marwan, N., Carmen Romano, M., Thiel, M., Kurths, J.: Recurrence plots for the analysis of complex systems. Physics Reports 438(5), 237-329 (2007). https://doi.org/10.1016/j.physrep.2006.11.001

[28] MATLAB: Version 2014a. The MathWorks Inc., Natick, Massachusetts (2014)

[29] Gomes, E.F., Jorge, A.M., Azevedo, P.J.: Classifying heart sounds using peak location for segmentation and feature construction. In: Proceedings of the International C* Conference on Computer Science and Software Engineering, pp. 23-30. ACM, ??? (2013)

[30] López Pérez, D., Bokde, A.L.W., Kerskens, C.: Cardiac related complexity changes of zero-quantum coherence (zqc) signals in brain $\mathrm{mr}$ imaging: Comparison between young and older healthy volunteers. bioRxiv, 20200527117226 (2020). https://doi.org/10.1101/2020.05.27.117226 
[31] Inc., W.R.: Mathematica, Version 12.3.1. Champaign, IL, 2021. https: //www.wolfram.com/mathematica

[32] Dagli, M.S., Ingeholm, J.E., Haxby, J.V.: Localization of cardiac-induced signal change in fmri. NeuroImage 9(4), 407-415 (1999). https://doi.org/ 10.1006/nimg.1998.0424

[33] López Pérez, D.: Private communication (2015)

[34] Baum, J., Munowitz, M., Garroway, A.N., Pines, A.: Multiple-quantum dynamics in solid state NMR. Multiple-quantum dynamics in solid state NMR,J. Chem. Phys. 83, 2015 (1985) (1985)

[35] Nakamura, T., Miyati, T., Kasai, H., Ohno, N., Yamada, M., Mase, M., Hara, M., Shibamoto, Y., Suzuki, Y., Ichikawa, K.: Bulk motionindependent analyses of water diffusion changes in the brain during the cardiac cycle. Radiological Physics and Technology 2(2), 133-137 (2009). https://doi.org/10.1007/s12194-009-0056-3

[36] Warren, W.S., Ahn, S., Mescher, M., Garwood, M., Ugurbil, K., Richter, W., Rizi, R.R., Hopkins, J., Leigh, J.S.: MR imaging contrast enhancement based on intermolecular zero quantum coherences. Science 281(5374), 247-251 (1998)

[37] Rizi, R.R., Ahn, S., Alsop, D.C., Garrett-Roe, S., Mescher, M., Richter, W., Schnall, M.D., Leigh, J.S., Warren, W.S.: Intermolecular zero-quantum coherence imaging of the human brain. Magnetic Resonance in Medicine 43(5), 627-632 (2000). https://doi.org/10.1002/(sici) 1522-2594(200005)43:5〈627::aid-mrm2 $\rangle 3.0 . c 0 ; 2-$ j

[38] Park, H.-D., Blanke, O.: Heartbeat-evoked cortical responses: Underlying mechanisms, functional roles, and methodological considerations. NeuroImage 197, 502-511 (2019). https://doi.org/10.1016/j.neuroimage. 2019.04 .081

[39] Kerskens, C.: Revisiting the quantum brain. Preprints (2020030459) (2020). https://doi.org/10.20944/preprints202003.0459.v1 


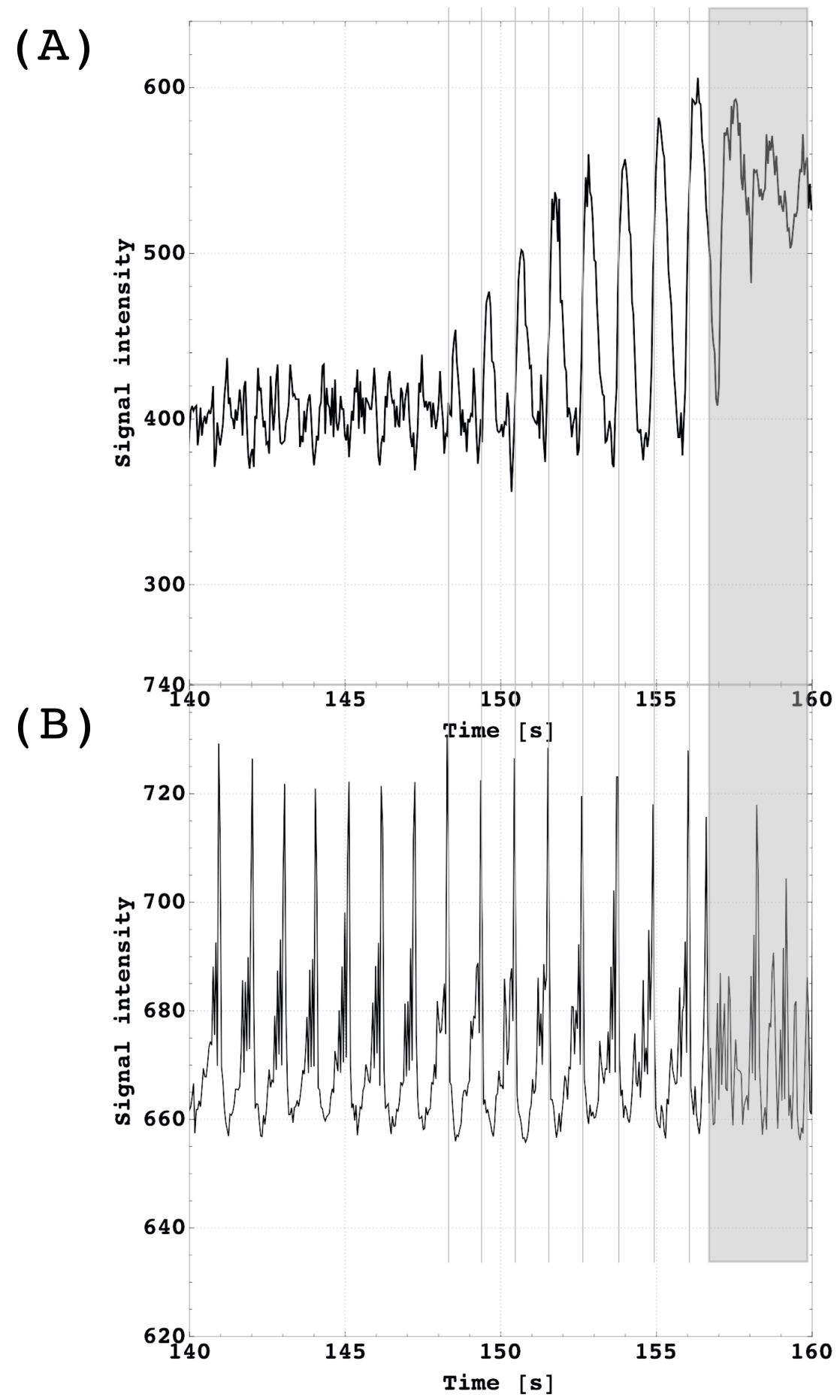

Fig. 4 (A) Signal time course of an imaging voxel located next to the superior sagittal sinus demonstrates the blood flow increase in response to the $\mathrm{CO}_{2}$ challenge (breath-holding). In contrast to the vein signal, the corresponding ZQC signals (B) showed no response to $\mathrm{CO}_{2}$ activity. Breath-holding started at 140s. Volunteers were instructed to reduce any movement as long as possible (here until at $157 \mathrm{~s}$ ). From $157 \mathrm{~s}$, the signal breakdown was subject to movement. 


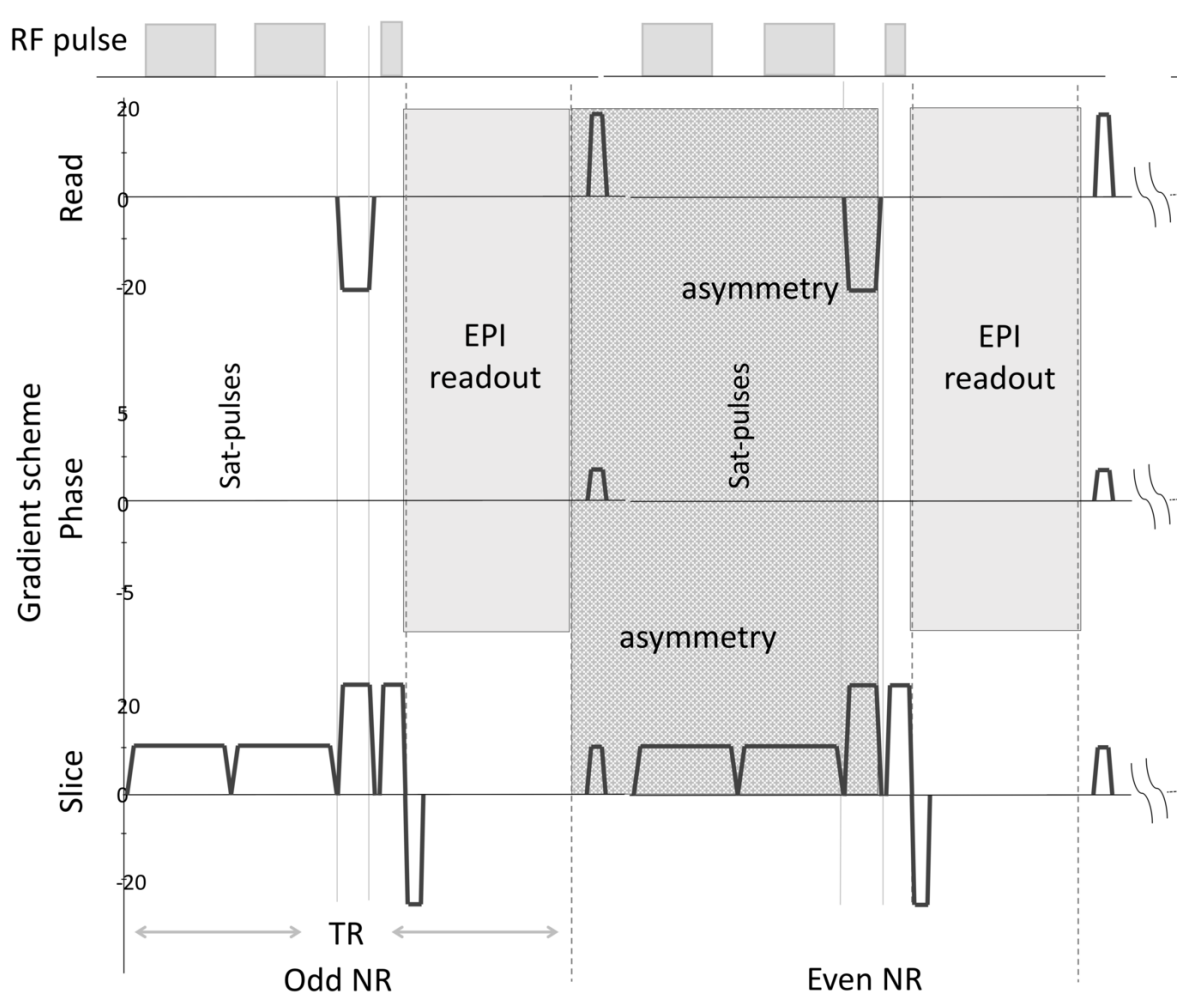

Fig. 5 Radio frequency (RF) and Gradient scheme of two consecutive EPI acquisition. The "asymmetry" field includes all asymmetric gradients involved in the ZSE.

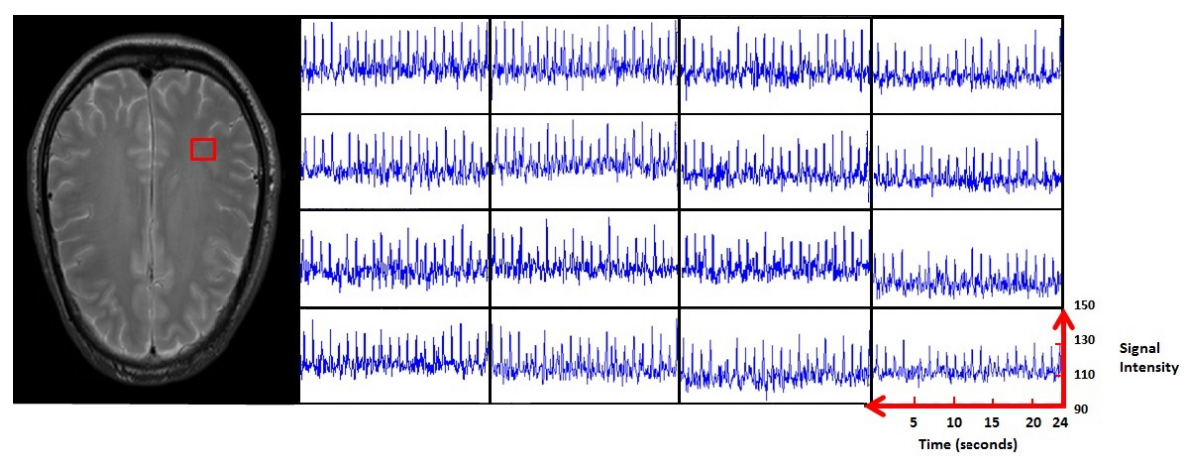

Fig. A1 4x4 voxel matrix randomly picked. On the left, the red square shows location in the brain slice. On the right, 16 corresponding signal time courses displaying the local tissue responses over a time period of $24 \mathrm{~s}$. 

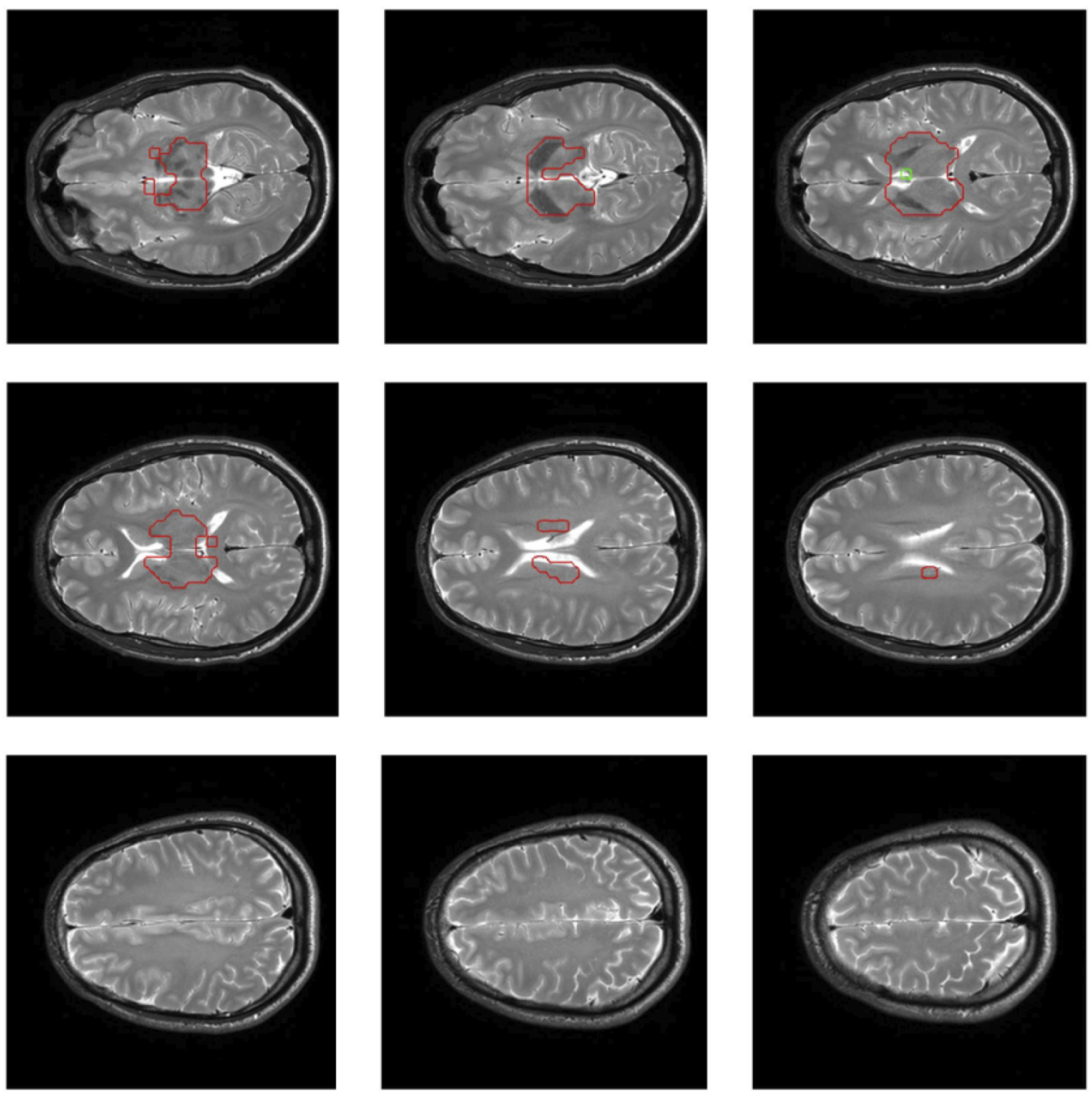

Fig. A2 9 Anatomical slices which correspond to the positioning of the EPI time series. Tissue surrounded by red drawing showed no ZQC bursts.

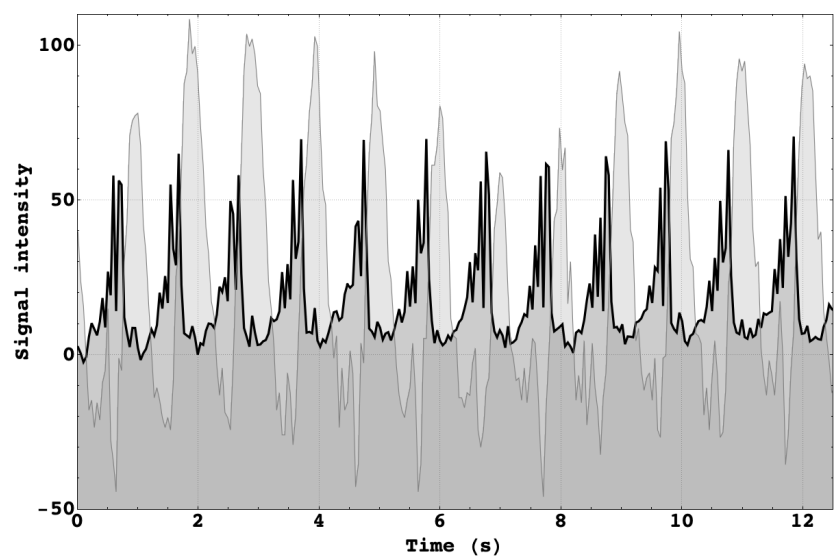

Fig. A3 Whole-slice averaged signal time course (black line) which was selected by a mask over 12 heart cycles. Signal of the Superior sagittal sinus (grey line) as reference time frame demonstrates the instant breakdown of quantum coherence with the beginning outflow. 
(A)

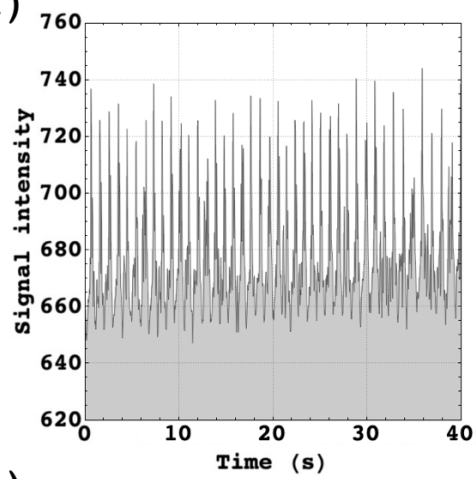

(C)

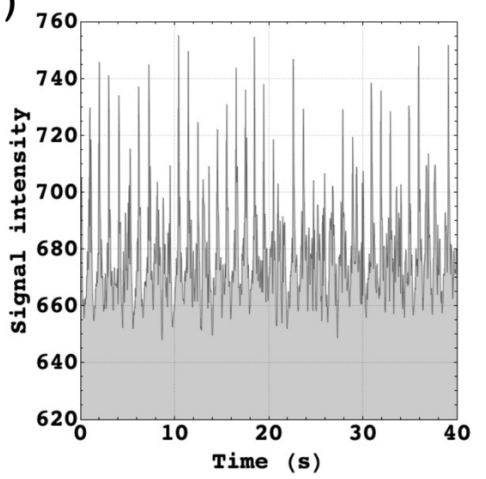

( $\mathrm{E})$

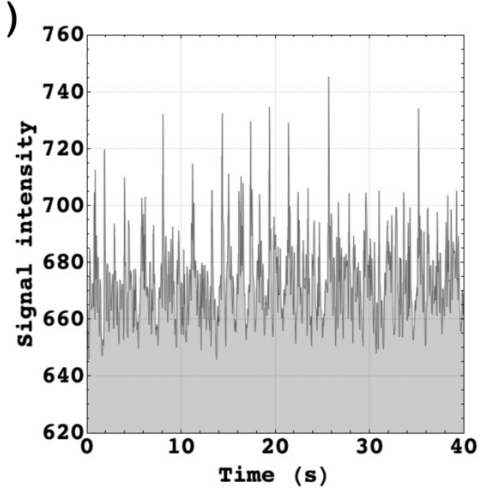

(B)

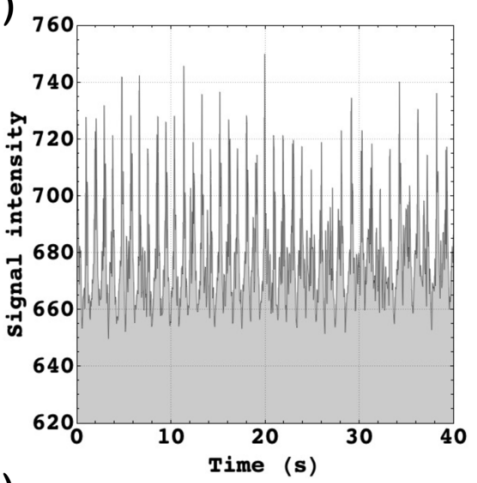

(D)

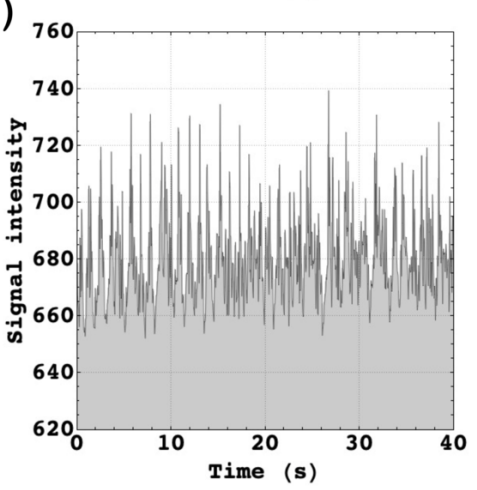

( $F)$

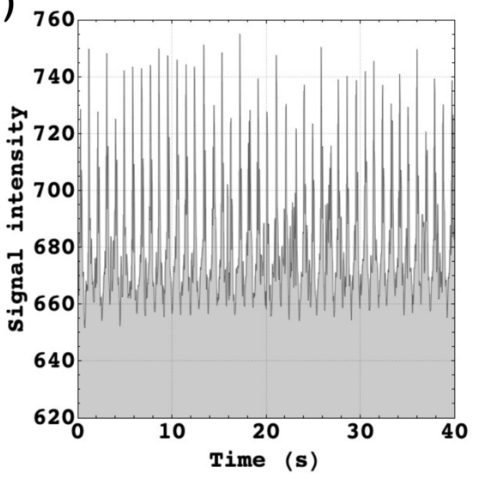

Fig. A4 Case study: ZQC burst pattern observed in participant who had reported falling asleep. Starting point of time series at (A) 16:26:29 (B) 16:29:47 (C) 16:30:54 (D) 16:34:13 (E) 16:37:32 (F) 16:40:49 (awake, subject communicated with radiographer before scan). 

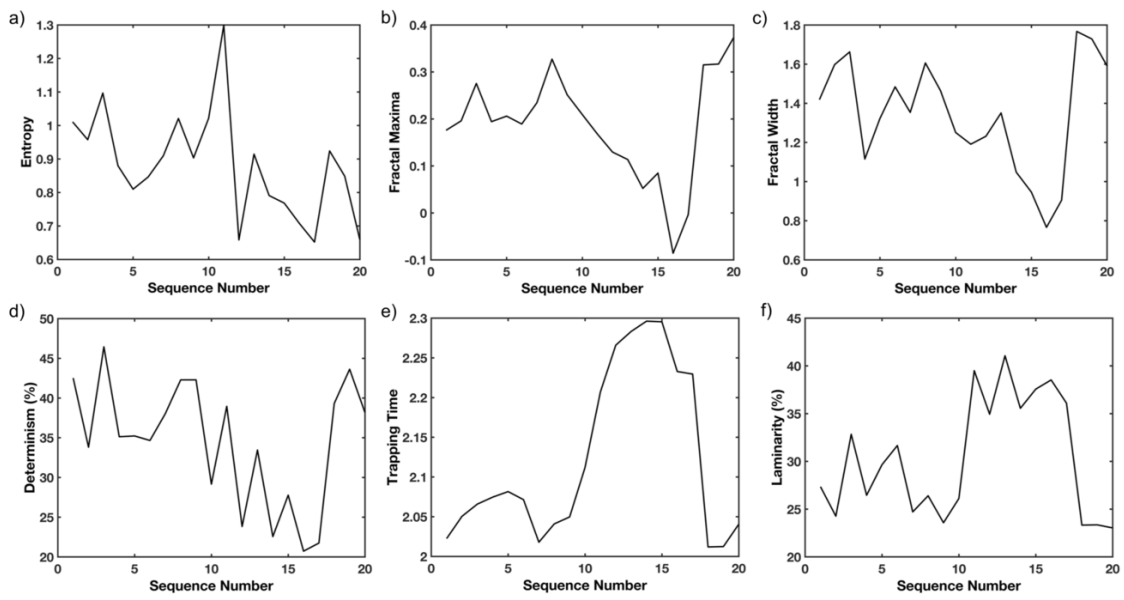

Fig. A5 Case study: Results of a Recurrence Quantication Analysis and a Multifractal Detrended Fluctuation Analysis using 20 time periods a 45s over a total time period of 21 minutes. (a) Entropy (Ent) is computed as the Shannon entropy of the distribution of the repeating pattern of the system. If a signal has high entropy it exhibits diversity in short and long duration periodicities. (b-c) The multifractal spectrum identifies the deviations in fractal structure within time periods with large and small fluctuations. (d) Determinism (DET) represents a measure that quantifies repeating patterns in a system and it is a measure of its predictability. Regular, periodic signals, such as sine waves, will have higher DET values, while uncorrelated time series will cause low DET. (e) Trapping Time (TT) represents the average time the system remains in a given state and it is a measure of the stability of the system. (f) Laminarity (Lam) determines the frequency of transitions from one state to another, without describing the length of these transition phases. It indexes the general level of persistence in some particular state of one of the time-series. 